\title{
Mini Nutritional Assessment (MNA) como método de evaluación nutricional en pacientes hospitalizados
}

\author{
O. IZAOLA ${ }^{1,2}$, D.A. DE LUIS ROMÁN ${ }^{1,2}$, G. CABEZAS ${ }^{1}$, S. ROJO ${ }^{1,2}$, L. CUÉLLAR ${ }^{2}$, \\ M.C. TERROBA ${ }^{1,2}$, R. ALLER ${ }^{2,3}$, M. GONZÁLEZ SAGRADO ${ }^{1,2}$ \\ ${ }^{1}$ Unidad de Apoyo a la Investigación. Sección de Endocrinología y Nutrición Clínica. \\ Hospital Universitario del Río Hortega. ${ }^{2}$ Instituto de Endocrinología y Nutrición Clínica. \\ Facultad de Medicina. ${ }^{3}$ Servicio de Digestivo. Hospital Clínico Universitario. Valladolid
}

\section{RESUMEN}

Objetivo: El objetivo del trabajo fue identificar la prevalencia de la desnutrición en los pacientes hospitalizados atendidos por nuestra Unidad utilizando el test MNA (Mini Nutritional Assessment) como herramienta de estudio.

Métodos: Estudiamos un total de 145 pacientes ingresados en el Hospital del Río Hortega, desde marzo de 2000 a mayo de 2002. A todos ellos se les realizó el test MNA, valoración antropométrica y bioquímica.

Resultados: La edad media de los pacientes fue de 57,44 $\pm 18,06$ años, el peso $63,5 \pm 13,7 \mathrm{~kg}$ y el índice de masa corporal $23,4 \pm 4,9$ $\mathrm{kg} / \mathrm{m}^{2}$. La población estudiada presentaban tumores hematológicos o en otras localizaciones en un $48,6 \%$, frente al resto de los pacientes que presentaban accidentes cerebrovasculares $21 \%$, problemas respiratorios o infecciones $16,8 \%$, insuficiencia renal $3,5 \%$ y enfermedades neurológicas o procesos de demencia en menor proporción $2,9 \%$.

Se dividió a los pacientes en dos grupos, los que presentaban desnutrición MNA $<17$ ( $\mathrm{n}=99)$ (grupo I) y los que presentaban riesgo nutricional MNA $\geq 17(\mathrm{n}=43$ ) (grupo II). La frecuencia de desnutrición fue del $68,2 \%$ (grupo I) y de riesgo de desnutrición del 29,6\% (grupo II) con el test MNA; siendo la puntuación media de 14,55 \pm 4,4 puntos.

En la valoración bioquímica el grupo de pacientes con desnutrición (grupo I) tuvo menores valores de albúmina $(2,9 \pm 0,7 \mathrm{~g} / \mathrm{dl} v s 3,41 \pm 2,71$ $\mathrm{g} / \mathrm{dl} ; \mathrm{p}<0,05)$, prealbumina $(14,9 \pm 6,2 \mathrm{~g} / \mathrm{dl}$ vs $17,6 \pm 8,1 \mathrm{~g} / \mathrm{dl} ; \mathrm{p}<0,05)$ y transferrina $(152,2 \pm 54,9 \mathrm{~g} / \mathrm{dl} v s 189,9 \pm 32,6 \mathrm{~g} / \mathrm{dl} ; \mathrm{p}<0,05)$, el resto de parámetros se mostraron dentro de la normalidad. En el análisis de los valores antropométricos la circunferencia muscular del brazo y la circunferencia del brazo se encontraron por debajo de la media en el grupo de pacientes con desnutrición (grupo I), no así el pliegue tricipital, el peso y el índice de masa corporal (IMC).

$\mathrm{Al}$ analizar los distintos apartados del test MNA entre ambos grupos, se observó como en el grupo de pacientes desnutridos la puntuación se encontraba por debajo de la media en todos los aportados.

La estancia media fue más elevada, aunque no se alcanzaron diferencias significativas en el grupo de pacientes desnutridos $(29,5 \pm 20$ días frente a $23,7 \pm 18$ días; $\mathrm{p}>0,05$ ).

Conclusiones: Los pacientes hospitalizados presentaron un riesgo de desnutrición elevado, detectado por el test MNA.

PALABRAS CLAVE: Desnutrición hospitalaria. Estado nutricional. Mini nutricional test.
MINI NUTRITIONAL ASSESSMENT (MNA) TEST AS A TOOL OF NUTRITIONAL EVALUATION IN HOSPITALIZED PATIENTS

\section{ABSTRACT}

Objetive: The objetive of the study was to identified the prevalence of malnutrition in come patients with MNA (Mini Nutritional Assessment) test as a clinical tool.

Methods: A total of 145 patients were studied in Hospital del Río Hortega (Valladolid), from mars of 2000 till may of 2002. In all patients a MNA test, an anthropometric and biochemical evaluation were performed.

Results: Mean age was $57.44 \pm 18.06$ years, weight $63.5 \pm 13.7 \mathrm{~kg}$ and body mass index $23.4 \pm 4.9 \mathrm{~kg} / \mathrm{m}_{2}$. The studied population were $48.6 \%$ haematological tumours and other tumours, opposite to the rest of the patients who were presenting cerebro-vascular accidents $2.1 \%$, respiratory problems or infections $16.8 \%$, renal failure $3.5 \%$ and neurological diseases or processes of dementia in minor proportion $2.9 \%$.

Patients were divided in two groups, malnutrition MNA $<17(n=$ 99) (group I) and risk of malnutrition $M N A \geq 17(n=43)$ (group II). The malnutrition prevalence was $68.2 \%$ (group I) and risk of malnutrition $29.6 \%$ (group II) with test MNA.

In biochemical evaluation the malnutrition group I had low levels of albumin $(2.9 \pm 0.7 \mathrm{~g} / \mathrm{dl}$ vs $3.41 \pm 2.71 \mathrm{~g} / \mathrm{dl} ; p<0.05)$, prealbumin $(14.9 \pm$ $6.2 \mathrm{~g} / \mathrm{dl}$ vs $17.6 \pm 8.1 \mathrm{~g} / \mathrm{dl} ; p<0.05)$ and transferrin $(152.2 \pm 54.9 \mathrm{~g} / \mathrm{dl} \mathrm{vs}$ $189.9 \pm 32.6 \mathrm{~g} / \mathrm{dl} ; p<0.05$. Arm muscular circumference and arm circumferce had values under average in patients with malnutrition.

On having analyzed the different paragraphs of the test MNA between both groups, it was observed since in the group of undernourished patients the punctuation he was below the average in all the paragraphs.

Conclusions: The risk of malnutrition in hospital was frequent, detected by MNA test.

KEY WORDS: Malnutrition in hospital. Nutritional status. Mini nutritional test.

Izaola O, de Luis Román DA, Cabezas G, Rojo S, Cuéllar L, Terroba MC, Aller R, González Sagrado M. Mini Nutritional Assessment (MNA) como método de evaluación nutricional en pacientes hospitalizados. An Med Interna (Madrid) 2005; 22: 313-316.

Trabajo aceptado: 14 de marzo de 2005 


\section{INTRODUCCIÓN}

El estado de salud de un individuo está a menudo comprometido por un estado nutricional deficiente (desnutrición), manifestándose clínicamente mediante pruebas bioquímicas y antropométricas (1).

La implantación de un programa de valoración nutricional en el ámbito hospitalario facilita la detección precoz de pacientes con desnutrición o riesgo y por consiguiente la instauración de medidas nutricionales para una mejor calidad de vida. Diversas investigaciones han demostrado que la valoración nutricional debe formar parte integral de la evaluación clínica de los pacientes con enfermedades crónicas y/o ancianos que requieren un soporte nutricional para disminuir los riesgos de morbimortalidad secundaria a la desnutrición (2).

Se disponen de un cierto número de instrumentos de cribaje que ayudan a evaluar el estado nutricional como es el caso del índice de masa corporal (IMC), pérdida de peso, pliegue cutáneo tricipital (PT), circunferencia braquial (CB), evaluación global subjetiva (SGA), índice pronóstico nutricional (IPN), índice de probabilidad nutricional (IMP), índice de riesgo nutricional (IRN) y evaluación del estado nutricional (MNA) (3).

El objetivo del presente estudio fue determinar la frecuencia de la desnutrición utilizando el MNA y valorar si existe relación entre los parámetros de IMC, pliegue tricipital, circunferencia del brazo con este test.

\section{MATERIAL Y MÉTODOS}

\section{PACIENTES}

Se estudiaron un total de 145 pacientes hospitalizados en el Hospital Universitario Río Hortega desde marzo de 2000 a mayo de 2002 (muestreo no probabilístico, consecutivo). Estos pacientes procedían de un área rural y urbana de Valladolid.

A todos los pacientes se les tomaron los siguientes datos epidemiológicos: edad, sexo, patología de hospitalización, realizándose una valoración antropométrica y bioquímica, así como el test MNA.

\section{VALORACIÓN ANTROPOMÉTRICA}

Se les pesó en una báscula calibrada cada 100 gramos y midió con un tallímetro estándar para posteriormente calcular el índice de masa corporal (IMC) con la fórmula (peso/talla2) $(\mathrm{kg} / \mathrm{m} 2)$.

Así mismo, se les midió el pliegue tricipital (PT). Para ello se mantuvo al paciente de pie y con su brazo no dominante colgado libremente. A continuación se localizó en la cara posterior el punto medio entre el acromio y el olécranon, y se pellizco suavemente la piel y el tejido celular subcutáneo en este punto y se aplicó perpendicularmente un calibrador regulado a presión $\left(10 \mathrm{~g} / \mathrm{mm}^{2}\right)$ durante 3 segundos (tipo Langer). La medición se realizó 3 veces y se tomó la media de estas, expresada en milímetros.

También se determinó la circunferencia del brazo (CB) y la circunferencia muscular del brazo (CMB). Para determinar estos parámetros se midió la $\mathrm{CB}$ con una cinta métrica calibra- da en milímetros en el mismo lugar del brazo donde se realizó la medición del PT.

Para el cálculo final de la CMB se utilizó la fórmula $(\mathrm{CMB}=\mathrm{CB}-(\mathrm{PT} \times 0,0314))$, expresado en milímetros.

Todos estos parámetros fueron medidos por la misma persona para evitar la variabilidad interindividual en las medidas antropométricas. Se utilizaron como tablas de referencia las de Frisancho y cols. (4).

\section{TEST MNA}

A todos los pacientes se les realizó al ingreso el test MNA, para evaluar el estado nutricional. Es un método validado y relativamente sencillo de utilizar, mediante 18 preguntas breves divididas en cuatro bloques. El primero bloque hace referencia a las mediciones antropométricas (índice de masa corporal, circunferencia braquial, circunferencia de la pierna y pérdida de peso), en el segundo bloque corresponde a la evaluación global del paciente con 6 preguntas sobre el estilo de vida, medicación y movilidad, a continuación se hace referencia a la evaluación nutricional mediante las preguntas de número de comidas, ingesta de alimentos, líquidos y autonomía para comer y ya por último se realiza una evaluación subjetiva del estado nutricional mediante la autoevaluación del estado nutricional y comparación con otras personas de su misma edad $(5,6)$.

La puntuación obtenida (máximo 30 puntos) permite clasificar a los pacientes en: estado nutricional satisfactorio (24 puntos), riesgo nutricional de malnutrición (entre 23,5 y 17 puntos) y con mal estado nutricional, es decir desnutrición a todos aquellos que se encuentren por debajo de 17 puntos.

\section{ANÁLISIS ESTADÍSTICO}

Con todos los datos obtenidos se creó una base de datos con el paquete estadístico (SPSS. Inc. IL., USA). Se realizó un análisis descriptivo de las variables cuantitativas, expresadas como (media \pm desviación estándar) y se compararon mediante el test de t Student. También se realizó una distribución de las variables en percentiles para estudiar el grado de desviación sobre la normalidad de estos pacientes.

\section{RESULTADOS}

Se estudiaron un total de 145 pacientes (60 varones y 85 mujeres) con una edad media de $57,44 \pm 18$ años, el peso 63,5 $\pm 13,7 \mathrm{~kg}$ y el índice de masa corporal $23,4 \pm 4,9$. El 48,6\% de la población estudiada presentaban tumores hematológicos o en otras localizaciones, frente al resto de los pacientes que presentaban accidentes cerebrovasculares $2,1 \%$, problemas respiratorios o infecciones $16,8 \%$, Insuficiencia renal 3,5\% y enfermedades neurológicas o procesos de demencia en menor proporción 2,9\%. La estancia media de estos pacientes en el hospital fue de 27,54 $\pm 20,07$ días.

La frecuencia de desnutrición fue del 68,2\% y de riesgo de desnutrición del 29,6\% con el test MNA; sin embargo, solamente 3 pacientes del total presentaban un buen estado nutricional.

Para el análisis de los resultados del MNA dividimos a los 
pacientes en dos grupos, los que presentaban desnutrición MNA $<17(\mathrm{n}=99)$ (grupo I) y los que presentaban riesgo de desnutrición MNA $\geq 17$ ( $\mathrm{n}=43$ ) (grupo II).

La valoración bioquímica de ambos grupos se muestra en la tabla I, observándose una depleción del compartimento proteico visceral. El grupo de pacientes con desnutrición (grupo I) tuvo menores valores de albúmina $(2,9 \pm 0,7 \mathrm{~g} / \mathrm{dl} v s 3,41 \pm$ $2,71 \mathrm{~g} / \mathrm{dl} ; \mathrm{p}<0,05)$, prealbumina $(14,9 \pm 6,2 \mathrm{~g} / \mathrm{dl}$ vs $17,6 \pm 8,1$ $\mathrm{g} / \mathrm{dl} ; \mathrm{p}<0,05)$ y transferrina $(152,2 \pm 54,9 \mathrm{~g} / \mathrm{dl}$ vs $189,9 \pm 32,6$ $\mathrm{g} / \mathrm{dl} ; \mathrm{p}<0,05)$ que el grupo con riesgo de desnutrición (grupo II). El resto de parámetros se mostraron dentro de la normalidad, aunque un $38,6 \%$ de los pacientes presentaron valores de glucosa por encima de $110 \mathrm{mg} / \mathrm{dl}$ (alteración de la glucemia en ayunas).

TABLA I

\begin{tabular}{|c|c|c|}
\hline \multicolumn{3}{|c|}{ VALORACIÓN BIOQUÍMICA } \\
\hline & $\begin{array}{c}\text { Desnutrición } \\
\text { Grupo I } \\
(n=99)\end{array}$ & $\begin{array}{c}\text { Riesgo desnutrición } \\
\text { Grupo II } \\
(n=43)\end{array}$ \\
\hline Linfocitos (103/uL) & $1.259,9 \pm 1.179,6$ & $1.289,2 \pm 849,19$ \\
\hline Glucosa (mg/dl) & $110,7 \pm 35,35$ & $110,7 \pm 35,35$ \\
\hline Urea (mg/dl) & $52,26 \pm 41,86$ & $37,41 \pm 24,50$ \\
\hline Creatinina (mg/dl) & $1,32 \pm 1,13$ & $1,07 \pm 0,54$ \\
\hline Colesterol total (mg/dl) & $147,29 \pm 52,31$ & $165,30 \pm 51,69$ \\
\hline Triglicéridos (mg/dl) & $121,43 \pm 76,64$ & $121,63 \pm 75,28$ \\
\hline Albúmina $(\mathrm{g} / \mathrm{dl})$ & $2,9 \pm 0,73^{*}$ & $3,41 \pm 0,57$ \\
\hline Transferrina (mg/dl) & $152,2 \pm 54,93^{*}$ & $189,9 \pm 32,66$ \\
\hline Prealbumina (mg/dl) & $14,9 \pm 6,2^{*}$ & $17,6 \pm 8,12$ \\
\hline Proteínas totales (g/dl) & $5,9 \pm 1,09$ & $6,2 \pm 0,80$ \\
\hline
\end{tabular}

${ }^{*} p<0,005 *$ diferencias significativas.

El análisis de los valores antropométricos (Tabla II) del grupo de pacientes con desnutrición (grupo I) frente al de riesgo de desnutrición (grupo II) mostró unos valores de PT (11,4 $\pm 6,6 \mathrm{~mm}$ vs 17,5 $\pm 8,2 \mathrm{~mm} ; \mathrm{p}<0,05), \mathrm{CB}(24,3 \pm 4 \mathrm{~mm}$ vs $29,4 \pm 4,3 \mathrm{~mm} ; \mathrm{p}<0,05)$ y $\mathrm{CMB}(20,7 \pm 3 \mathrm{~mm}$ vs $23,9 \pm 3,1$ $\mathrm{mm} ; \mathrm{p}<0,05)$. Al realizar la distribución de estos tres parámetros antropométricos en función de los percentiles descritos por las tablas de referencia de Frisancho y cols. (3) y comparando los pacientes con riesgo de desnutrición (grupo II) frente a los de desnutrición (grupo I), se detectó como el (grupo II $46,2 \%$ vs grupo I 79,6\%; $\mathrm{p}<0,05)$ de los pacientes presenta-

\begin{tabular}{|c|c|c|}
\hline \multicolumn{3}{|c|}{ TABLA II } \\
\hline \multicolumn{3}{|c|}{ VALORACIÓN ANTROPOMÉTRICA } \\
\hline & $\begin{array}{l}\text { Desnutrición } \\
\text { Grupo I } \\
(n=99)\end{array}$ & $\begin{array}{c}\text { Riesgo desnutrición } \\
\text { Grupo II } \\
(n=43)\end{array}$ \\
\hline IMC & $21,97 \pm 4,47^{*}$ & $26,45 \pm 4,73$ \\
\hline PESO (kg) & $59,56 \pm 12,15^{*}$ & $72,30 \pm 13,12$ \\
\hline PT (mm) & $11,4 \pm 6,6^{*}$ & $17,5 \pm 8,27$ \\
\hline $\mathrm{CB}(\mathrm{cm})$ & $24,3 \pm 4,01^{*}$ & $29,4 \pm 4,37$ \\
\hline $\mathrm{CMB}(\mathrm{cm})$ & $20,7 \pm 3,02 *$ & $23,90 \pm 3,19$ \\
\hline
\end{tabular}

PT: pliegue tricipital; $\mathrm{CB}$ : circunferencia del brazo; CMB: circunferencia muscular del brazo. ${ }^{*} p<0,005 *$ diferencias significativas. ban un percentil del PT inferior o igual al P50, un (grupo II $72 \%$ vs grupo I 90,8\%; $\mathrm{p}<0,05)$ presentaron un percentil de $\mathrm{CMB}$ inferior o igual al P50, al analizar la CB un (grupo II $69,6 \%$ vs grupo I $72,8 \%$; p < 0,05) de los pacientes tenía un percentil inferior o igual al P50 (Tabla III).

\section{TABLA III}

DISTRIBUCIÓN DE PERCENTILES DE VALORES ANTROPOMÉTRICOS SEGÚN TABLA DE REFERENCIA DE FRISANCHO Y COLS. (5)

\begin{tabular}{lcccrrr}
\hline & \multicolumn{2}{c}{ P. tricipital } & \multicolumn{2}{c}{ C. brazo } & \multicolumn{2}{c}{ C. muscular } \\
& & & & \multicolumn{3}{c}{ del brazo } \\
& $R D(\%)$ & $D(\%)$ & $R D(\%)$ & $D(\%)$ & $R D(\%)$ & $D(\%)$ \\
\hline P 5 & 6,8 & 27,2 & 18,6 & 57,5 & 18,6 & 57,5 \\
P 10 & 6,9 & 11,1 & 13,9 & 9,0 & 6,9 & 1,0 \\
P 25 & 6,9 & 16,6 & 11,6 & 16,1 & 18,6 & 19,9 \\
P 50 & 25,5 & 25,2 & 27,9 & 8,0 & 25,5 & 14,4 \\
P 75 & 20,9 & 14,1 & 18,6 & 4,0 & 13,9 & 6,0 \\
P 90 & 25,5 & 5,0 & 9,3 & 3,0 & 6,9 & 2,0 \\
P95 & 6,9 & 1,0 & 0,0 & 1,0 & 9,3 & 0,0 \\
\hline
\end{tabular}

$\mathrm{RD}=$ riesgo desnutrición; $\mathrm{D}=$ desnutrición.

Al analizar los distintos apartados del test MNA (Tabla IV) y comparar los resultados entres los dos grupos, se observó como en el grupo de pacientes desnutridos la puntuación se encontraba por debajo de la media en todos los aportados, dando lugar a una puntuación media del test en los pacientes con desnutrición muy por debajo del punto de corte del test (MNA <17). La puntuación en todos los apartados fue significativamente inferior en el grupo de desnutridos .

Así mismo los pacientes con desnutrición presentaron una estancia media más elevada, aunque no se alcanzaron diferencias significativas $(29,5 \pm 20$ días frente a 23,7 \pm 18 días; $\mathrm{p}>$ $0,05)$.

\begin{tabular}{lccc}
\multicolumn{3}{c}{ TABLA IV } \\
& P. max & $\begin{array}{c}\text { Desnutridos } \\
\text { Grupo I } \\
(n=99)\end{array}$ & $\begin{array}{c}\text { R. desnutrición } \\
\text { Grupo II } \\
(n=43)\end{array}$ \\
\hline Indice antropométrico & 8 & $3,53 \pm 2,55^{*}$ & $6,40 \pm 1,63$ \\
Evaluación global & 8 & $3,91 \pm 1,48^{*}$ & $5,18 \pm 1,0$ \\
Parámetros dietéticos & 10 & $3,41 \pm 2,17^{*}$ & $5,69 \pm 1,85$ \\
Evaluación subjetiva & 4 & $1,57 \pm 0,77^{*}$ & $2,18 \pm 0,66$ \\
Total test MNA & 30 & $12,43 \pm 3,46^{*}$ & $19,43 \pm 1,51$ \\
\hline
\end{tabular}

P. Max: puntuación máxima; ${ }^{*} p<0,005 *$ diferencias significativas.

DISCUSIÓN

La valoración nutricional de un individuo está íntimamente relacionado con el estado de salud, debiéndose incluir en la historia clínica y dietética de cada paciente. Entre un 30 y un 70 por ciento de los pacientes hospitalizados presentan desnutrición, repercutiendo negativamente en la evolución del enfermo e incrementando el riesgo de fallecimiento. 
Las reservas energéticas del tejido adiposo o de la masa grasa (MG) y de la masa magra están estrechamente relacionadas con la masa metabólica o celular del organismo encargado de mantener las funciones vitales (7). En situación de enfermedad se produce modificaciones en ambos compartimentos. Para determinarlos se emplean medidas antropométricas y parámetros bioquímicas con el fin de identificar a los pacientes "malnutridos".

Existen diversos instrumentos de cribaje que ayudan a evaluar el estado nutricional como es el caso del test MNA, el cual en diversas investigaciones ha demostrado que permite anticiparse a la aparición de otros marcadores bioquímicos de malnutrición detectando una población de riesgo antes de que la respuesta inmunitaria se altere (9).

Los estudios que existen en la literatura que correlacionan el test MNA con el estado nutricional se han centrado en pacientes de tercera edad (10-12). En el caso de ancianos hospitalizados, el rango de malnutrición proteico calórica oscila desde el 18 al 65\% (10). En un estudio de 1.300 sujetos ancianos hospitalizados, entre el 40 y el $55 \%$ estaban malnutridos o en riesgo de malnutrición, mientras que el $12 \%$ estaban afectados por una malnutrición grave (13), esto es debido a que el estado nutricional de estos pacientes está asociado con enfermedades crónicas sobre todo de tipo respiratorio y metabólico.

En la actualidad se está empezando a dar gran relevancia al estado nutricional del paciente antes del ingreso hospitalario $(14,15)$, debido a la clara relación que existe entre la malnutrición calórico-proteica, complicaciones, estancia hospitalaria y gasto sanitario. En nuestro trabajo un alto número de pacientes presentaron una depleción tanto del compartimento graso como del magro. Los resultados analizados mediante los parámetros antropométricos demostraron que un $54,9 \%$ de los pacientes se encontraban desnutridos $(\mathrm{p}<25)$ valor similar al obtenido mediante el test MNA con un $68,2 \%$.

Así mismo, los pacientes oncológicos son considerados también como población de mayor riesgo de desnutrición, como se ha podido corroborar en el estudio de Edington et al., que encontró un $10 \%$ de desnutrición en pacientes oncológicos y un $8 \%$ en pacientes con enfermedades crónicas que residían en su domicilio (16), como consecuencia de un aumento de las necesidades de energía y nutrientes en un momento en el que la ingesta está reducida por la enfermedad (17). En nuestro estudio esta población representa un 48,6\% correlacionado con el test MNA.

El coste potencial de la malnutrición es elevado, sin embargo queda demostrado una vez más que con una herramienta sencilla y barata como es el test MNA podemos diagnosticar un paciente desnutrido o con riesgo de desnutrición para realizar una intervención nutricional que supondrá una menor estancia hospitalaria, por consiguiente un menor gasto sanitario y en definitiva una mejor calidad de vida para los pacientes (18-20).

En conclusión, los pacientes vistos en nuestro hospital presentaron un riesgo de desnutrición elevado, correlacionado tanto por los parámetros bioquímicos, antropométricos y el test MNA. Aunque no existe un método ideal para la valoración del estado nutricional, si existen diversas herramientas de fácil acceso que se complementan.

\section{Bibliografía}

1. Casimiro C, García de Lorenzo A, et al. Estado Nutricional y metabólico y valoración dietética en pacientes ancianos, institucionalizados con diabetes mellitus no insulin dependiente. Nutr Hosp 2001; 16: 104-111.

2. Terán Estrada L, Calderón González MR, Castillo Pineda JC, et al. La valoración global subjetiva como método de evaluación nutricional en artritis reumatoide. Rev Mex Reumat 2002; 17: 117-122.

3. Guigoz Y, Vellas BJ, Garry PJ. Assessing the nurtitional status of the elderly. The Mini Nutritional Asssessment as part of the geriatric evaluation. Nutr Rev 1996; 54: 59-65.

4. Frisancho AR. New norms of upper limb fat and muscle areas for assessment of nutritional status. Am J Clin Nutri 1981; 34: 540-545.

5. Guigoz Y. Vellas B, Garry PJ. Assessing the nutritional status of the elderly: The Mini Nutritional Assesment as part of the geriatric evaluation. Nutrit Surv Elderly 1996; 54: S59-65.

6. Cohendy R, Rubenstein LZ, Eledjan J. The Mini Nutritional Assessment- Short Form for preoperative nutritional evaluation of elderly patients. Again 2001; 13: 293-7.

7. Martín Peña G. Valoración del estado nutricional.Nutrición en atención primaria. 2001: 43-54.

8. DA de Luis, R. Aller, P. Bachiller. Descripción del estado nutricional en una muestra de pacientes con infección VIH. Ana Med Interna (Madrid) 2001; 18: 619-623.

9. DA de Luis, R. Aller, P. Bachiller. Diferencias en la clasificacion antropométrica de pacientes con infección por VIH, con dos tablas de referencia. Nutr Clin Dietética 2000; 20: 29-34.

10. Schiffrin EJ, Guigoz Y, Perruisseau G, Delneste Y, Mansaurian R, et al. MNA e inmunidad: marcadores nutricionales e inmunológicos en las personas de edad avanzada. Nestlé Nutrition Workshop Series. 1999.
Vol 1: 7-9.

11. Soini H, Routasalo P, Lagstrom H. Characteristics of the Mini-Nutritional Assessment in elderly home-care patients. Eur J Clin Nutr 2004; 58: 64-70.

12. August DA. Creation of a specialized nutrition support outcomes rearch consortium: if not now, when? JPEN 1996; 20: 339-400.

13. Donini LM, Savina C, Rosano A, De Felice MR, Tassi L, De Bernardini L, Pinto A, Giusti AM, Cannella C. MNA predictive value in the follow-up of geriatric patients. J Nutr Health Aging 2003; 7: 282-93.

14. Persson MD, Brismar KE, Katzarski KS, Nordenstrom J, Cederholm TE. Nutritional status using mini nutritional assessment and subjective global assessment predict mortality in geriatric patients. J Am Geriatr Soc 2002; 50: 1996-2002.

15. Pirlich M, Schutz T, Kemps M, Luhman N, Burmester GR, Baumann G, et al. Prevalence of malnutrition in hospitalized medical patients: impact of underlying disease. Dig Dis 2003; 21: 245-51.

16. Pablo AM, Izaga MA, Alday LA. Assessment of nutritional status on hospital admission: nutritional scores. Eur J Clin Nutr 2003; 57: 824-31.

17. Vetta F, Ronzoni S, Palleschi L, Bollea MR. Multidimensional approach for nutritional evaluation and restore in the elderly. Clin Nutr 1997; 16: 269-270.

18. Edington J, Kon P, Martyn CN. Prevalence of malnutrition in patients in medical practice. Clin Nutr 1996; 15: 60-63.

19. Griep M, Mets TF, Collys K, Verte D, Verleye G, Massart D. MNA and odor perception. First Nutrition Workshop. Laussanne, 1997: 20-21.

20. Gallaghger-Allred CR, Voss AC, Finn SC, McCamish MA. Malnutr ition and clinical outcomes: the case for medical nutrition therapy. J Am Diet Assoc 1996, 96: 361-366. 